\title{
Migrant mental health, Hickam's dictum, and the dangers of oversimplification
}

\author{
Jordan Edwards ${ }^{1,4} \cdot$ Kelly K. Anderson ${ }^{1,2,4} \cdot$ Saverio Stranges ${ }^{1,3,5}$ \\ Received: 28 September 2018 / Accepted: 19 December 2018/ Published online: 11 January 2019 \\ (C) Swiss School of Public Health (SSPH+) 2019
}

The process of migration-including the reasons for migration, the migratory process, and post-migration resettlement-can cause extraordinary stress, which can influence mental well-being and rates of mental illness (Hollander 2013). In this editorial, we will discuss the challenges of addressing migrant mental health needs and highlight the shortcomings in the oversimplification of this issue.

\section{Challenges for health care systems}

There has been an increase in the global awareness of the public health relevance of mental disorders and poor mental well-being among migrant groups. This is highlighted by the current migrant crisis in Europe, where massive migrant influxes, many of whom are fleeing conflict ridden nations, are presenting with acute mental health care needs (Abbott 2016). These migrants and others with mental health needs pose numerous challenges for health care systems around the globe. Although barriers to accessing mental health services among migrant groups have decreased, there remain many gaps in mental health support (Close et al. 2016). Even among the most affluent

Jordan Edwards

jedwa@uwo.ca

1 Department of Epidemiology and Biostatistics, Schulich School of Medicine and Dentistry, The University of Western Ontario, London, ON, Canada

2 Department of Psychiatry, Schulich School of Medicine and Dentistry, The University of Western Ontario, London, ON, Canada

3 Department of Family Medicine, Schulich School of Medicine and Dentistry, The University of Western Ontario, London, ON, Canada

4 Lawson Health Research Institute, London, ON, Canada

5 Department of Population Health, Luxembourg Institute of Health, Strassen, Luxembourg nations, there remain challenges with mental health treatment for migrant groups, as cultural differences, language, discriminatory practices, health care coverage, and costs all influence help-seeking and health care utilization (Kirmayer et al. 2011; Priebe et al. 2016; Borraccino et al. 2018). These challenges are compounded in less affluent nations where a lack of health professionals and infrastructure, as well as limited mental health service investment and availability, leaves many mental health needs unaddressed (Kirkbride et al. 2017). Migrant health and health promotion initiatives have increased; however, evidence suggests that there has been an increase in the divide between policy and practice for migrant mental health (Zimmerman et al. 2011). There is a need for greater communication between policy-makers, researchers, and clinicians to develop context-specific practices to improve mental health outcomes for migrant groups (Zimmerman et al. 2011).

\section{Need for evidence}

Research on migrant mental health has increased over the past few decades, though there remain gaps in both health services and etiological research on the topic (Zimmerman et al. 2011). Although research aimed at improving health services typically requires a higher-level approach that ensures effective knowledge translation and implementation, there is conversely a need to expand research that adequately contextualizes various migrant groups to enable policy decision-makers to understand the heterogeneity that exists within these groups to further inform their work (Zimmerman et al. 2011). Areas of future research include the evaluation of novel strategies for providing adequate mental health care for migrant groups, with a particular emphasis on primary care settings (Kirmayer et al. 2011).

Research on the etiology of mental disorders and mental well-being requires a more detailed evaluation of the pathways that lead to negative mental health outcomes 
(Nesterko et al. 2018). There is a need for etiological research to explore differences by various immigrant and refugee groups, variation in risk factors, and to isolate the effects of specific migratory experiences.

Mental illness and mental well-being may represent two different but correlated means of assessing migrant mental health (Close et al. 2016). There is a need to compare these constructs and evaluate them. Another gap in the literature is the current lack of research assessing pre-migratory risk factors, as well as geographic variation, ethnic density, migrant generation, time since immigration, and post-migratory socioeconomic disadvantage, in relation to rates of mental illnesses and poor mental well-being among migrant groups (Khanlou and Jackson 2010).

\section{Hickam's dictum}

As a counterbalance to the more popular Occam's razor, which contends the simplest solution tends to be the right one, Hickam's dictum asserts that we should assume multiple causes for multiple outcomes, without unnecessary additional assumptions. Migrant mental health is a multi-dimensional topic with many internal and external influences; it is inherently a study of multiple causes. Researchers must recognize the inherent dangers of making broad assumptions when defining migrant groups, and the limitations of providing aggregate results when further detail is not available. When we delve into the examination of disparate migrant groups, who have vastly different migratory experiences, it is to be expected that mental health outcomes will diverge. For example, it is intuitive that there will be a large degree of dissimilarity between economic or family class migrant groups and refugee groups, though they are commonly grouped together (Kirmayer et al. 2011). This variation can entirely change ones conclusions of whether migrant status is a risk or protective factor for numerous mental illnesses (Kirmayer et al. 2011). Aggregate results may be drowning out opposing signals present in less prevalent migratory classes.

Researchers should recognize that the relationship between migration and mental health is dynamic and highly dependent on context, and the associated risk and protective factors. Where possible, researchers should differentiate between migrant groups to provide more focused results. Policy-makers will always be tasked with the challenge of striking a balance between specificity and feasibility of creating and implementing public health interventions. Nevertheless, more focused research will allow policy-makers to create more informed and sensitive mental health care policies for diverse migrant groups.
Migrant mental health is a global discussion. As such, we must make efforts in all areas of research and policy development to define outcomes in sufficient detail in order to promote research transparency and transferability, with an ultimate goal of more effectively addressing the mental health needs of all migrant groups.

\section{Compliance with ethical standards}

Conflict of interest The authors declare that they have no conflict of interest.

\section{References}

Abbott A (2016) The mental-health crisis among migrants. Nat News 538(7624): 158

Borraccino A, Charrier L, Berchialla P, Lazzeri G, Vieno A, Dalmasso P, Lemma P (2018) Perceived well-being in adolescent immigrants: it matters where they come from. Int J Public Health 63(9):1037-1045

Close C, Kouvonen A, Bosqui T, Patel K, O’Reilly D, Donnelly M (2016) The mental health and wellbeing of first generation migrants: a systematic-narrative review of reviews. Glob Health 12(1):47

Hollander AC (2013) Social inequalities in mental health and mortality among refugees and other immigrants to Swedenepidemiological studies of register data. Glob Health Action 6(1):21059

Khanlou N, Jackson A (2010) Immigrant mental health public health agency of Canada. http://www.metropolis.net/pdfs/immigrant mental_health_10aug10.pdf. Accessed 12 Sept 2018

Kirkbride JB, Hameed Y, Ioannidis K, Ankireddypalli G, Crane CM, Nasir M, Spyridi S (2017) Ethnic minority status, age-atimmigration and psychosis risk in rural environments: evidence from the SEPEA study. Schizophr Bull 43(6):1251-1261

Kirmayer LJ, Narasiah L, Munoz M, Rashid M, Ryder AG, Guzder J, Pottie K (2011) Common mental health problems in immigrants and refugees: general approach in primary care. CMAJ 183(12):E959-E967

Nesterko Y, Turrión CM, Friedrich M, Glaesmer H (2018) Trajectories of health-related quality of life in immigrants and nonimmigrants in Germany: a population-based longitudinal study. Int J Public Health. https://doi.org/10.1007/s00038-018-1113-7

Priebe S, Giacco D, El-Nagib R (2016). Public health aspects of mental health among migrants and refugees: a review of the evidence on mental health care for refugees, asylum seekers and irregular migrants in the WHO European Region. Health evidence synthesis report 47

Zimmerman C, Kiss L, Hossain M (2011) Migration and health: a framework for 21 st century policy-making. PLoS Med 8(5):e1001034

Publisher's Note Springer Nature remains neutral with regard to jurisdictional claims in published maps and institutional affiliations. 